\title{
Adhesion to type I collagen fibrous gels induces E- to N- cadherin switching without other EMT- related phenotypes in lung carcinoma cell A549
}

\author{
Hitomi Fujisaki ${ }^{1 *}$, Sugiko Futaki ${ }^{2}$, Masashi Yamada ${ }^{3}$, Kiyotoshi Sekiguchi ${ }^{3}$, Toshihiko Hayashi ${ }^{4,5}$, Shunji
} Hattori $^{1}$

${ }^{1}$ Nippi Research Institute of Biomatrix, Ibaraki, Japan

${ }^{2}$ Department of Anatomy and Cell Biology, Osaka Medical College, Osaka, Japan

${ }^{3}$ Division of Matrixome Research and Application, Institute for Protein Research, Osaka University, Osaka, Japan

${ }^{4}$ China-Japan Research Institute of Medical and Pharmaceutical Sciences, Wuya college of Innovation, Shenyang Pharmaceutical University, Shenyang, China

${ }^{5}$ Department of Chemistry Life Science, School Adv. Engineering, Kogakuin University, Tokyo, Japan

${ }^{*}$ Corresponding author: Hitomi Fujisaki

E-mail: fujisaki@nippi-inc.co.jp

Running title: Cell cluster via N-cadherin on Col-I gels

Keywords: Type I collagen, on-gel-culture, EMT, cancer line cell, cell cluster, cadherin switch 


\begin{abstract}
In culture system, environmental factors, such as increasing exogenous growth factors and adhesion to type I collagen (Col-I) induce epithelial-tomesenchymal transition (EMT) in cells. Col-I molecules maintain a non-fibril form under acidic conditions, and they reassemble into fibrils under physiological conditions. Col-I fibrils often assemble to form three-dimensional gels. The gels and non-gel-form of Col-I can be utilized as culture substrates and different gel-forming state often elicit different cell behaviors. However, gel-form dependent effects on cell behaviors, including EMT induction, remain unclear. EMT induction in lung cancer cell line A549 has been reported via adhesion to Col-I but the effects of gel form dependency are unelucidated. This study investigated the changes in EMT-related behaviors in A549 cells cultured on Col-I gels.

We examined cell morphology, proliferation, single-cell migration and expression of EMTrelated features in A549 cells cultured on gels or non-gel form of Col-I and non-treated dish with or without transforming growth factor (TGF)- $\beta 1$. On Col-I gels, some cells kept cell-cell contacts and formed clusters, others maintained single-cell form. In cell-cell contact regions, E-cadherin expression was downregulated, whereas that of $\mathrm{N}$-cadherin was upregulated. Vimentin and integrins $\alpha 2$ and $\beta 1$ expression were not increased. In TGF- $\beta 1$-treated A549 cells, cadherin switched from E- to Ncadherin. Their morphology changed to a mesenchymal form and cells scattered with no cluster formation. Vimentin, integrins $\alpha 2$ and $\beta 1$ expression were upregulated. Thus, we concluded that culture on Col-I fibrous gels induced E- to Ncadherin switching without other EMT-related phenotypes in A549 cells.
\end{abstract}

\section{Introduction}

Type I collagen (Col-I) is a ubiquitous and major component of interstitial tissues. In vivo, many kinds of cells are found resting proliferation within Col-I fibrils containing matrix, while, cellextracellular matrix (ECM) interactions are essential for cell movement (1). For a long while,
Col-I has been widely studied and used as a cell culture substrate (1-7). Typically, Col-I is solubilized under acidic conditions and extracted from animal tissues. Col-I remains in a non-fibril form under acidic conditions, meaning that Col-I molecules are not assembled each other. Soluble Col-I molecules reassemble into fibrils under physiological conditions (i.e., $\mathrm{pH}$, temperature, and ionic strength) spontaneously, and these fibrils often assemble into fiber bundles with a threedimensional structure (i.e., gels) (2-4). Col-I, as a culture equipment, has been roughly divided into two categories depending on whether or not gels are formed. In 1982, Kleinman et al. reported a few different Col-I treatment protocols for culture substrates, such as drying up acidic Col-I solution to cover the surface of a culture dish (or removing excess solution) and neutralizing acidic Col-I solution for gelation $(2,3)$. They called the former substrate "collagen films" and the latter "reconstituted collagen" (3). Sometimes, the former is called "2D culture substrate" and the latter is "3D culture substrate" respectively. Recently fibril-forming Col-I has been used as novel hydrogels (5) and the history of the collagen gel culture assay has been reviewed (6).

Adherence to different forms of Col-I (gels or nongel-form) elicits different cellular responses in various cells, such as morphological appearance and metabolic and proliferative potential. In fibroblast culture, there are differences between gels $(3,7)$ and non-gel-form $(3)$ in terms of the growth rates, mitogenic responses to growth factors, and collagen synthesis. Koyama et al. showed that arterial smooth muscle cells remained arrested in G1 phase in the presence of Col-I gels. However, when cultured on non-gel-form of Col-I, they proliferate (8). Furthermore, the culture of human keratinocytes on the non-gel form of Col-I results in the spread morphology and continuous growth. While, the culture on Col-I gels induces round morphology and apoptosis in cells (9). The level of reactive oxygen species (ROS) in 3T3-L1 murine preadipocytes increases on Col-I, independent of the gel-forming state, compared with on a non-treated dish. A markedly higher level of ROS is found in cells on Col-I gels, it serves as 
a suppressor of cell proliferation and migration. But higher level of ROS acts as a positive regulator in these processes on the non-gel-form of Col-I. These opposite effects of increasing ROS are attributed to the levels of nuclear factor- $\mathrm{\kappa B} / \mathrm{p} 65$ activation (10).

Epithelial-to-mesenchymal transition (EMT) is a fundamental biological process, whereby epithelial cells lose their apical-basal polarity, cell-cell adhesions via E-cadherin weaken, and the cells express mesenchymal cell characteristics, including $\mathrm{N}$-cadherin expression (11-13). Beside the switching of cadherin types, various EMTrelated markers (i.e., vimentin, matrix metalloproteinases, and transcription factors Snail, Slug, and Twist1) have been reported (12-15). The concept of EMT was proposed by Greenburg and Hay as a process induced by adherence to Col-I gels in embryonic chick corneal or lens epithelial cells (16). More recently, EMT has been associated with wound healing and cancer metastasis (17-20). Since the turn of the century, the number of papers on EMT has increased exponentially, and there are also more reports about intermediate cell behaviors between the epithelial and mesenchymal forms. The EMT International Association proposed naming the intermediate form, epithelial mesenchymal plasticity (EMP) (12). Many reports and discussions are ongoing regarding EMT and EMP. Various growth and differentiation factors can induce and regulate the EMT process. Among them, transforming growth factor (TGF) $-\beta$ is one of the well-characterized inducers or enhancers of EMT (21-24). Numerous signaling pathways that regulate or mediate the EMT process via the activation of TGF- $\beta$ receptors have been reported. Furthermore, increased exogenous TGF- $\beta$ upregulates ECM production and deposition, including Col-I, around the cellular microenvironment $(23,24)$. Col-I deposition has been attributed to increasing matrix stiffness. Additionally, TGF- $\beta 1$ signal activation contributes to a positive feedback autocrine loop in tumor cells undergoing EMT (25).

Adhesion to Col-I induces EMT-like upregulation of single-cell motility by regulating cellular signal transduction (26-29). Tiam1/Rac signaling promotes motility in Madin-Darby canine kidney cells cultured on Col-I, which is regulated by phosphatidylinositol 3-kinase (PI3K) pathway activation (26). Cells converted to highly invasive forms of pancreatic cancer are characterized by extensive Col-I deposition $(27,28)$. Shintani et al. showed that mouse mammary epithelial cells upregulated N-cadherin expression and underwent EMT in response to culture dishes coated with ColI through a pathway involving integrins and discoidin domain receptor tyrosine kinase 1 , including the activation of PI3K, Rac1, and c-Jun $\mathrm{N}$-terminal kinases. These effects are not induced with fibronectin or laminin (29). In the lung cancer cell line A549, EMT is induced via TGF- $\beta$ stimulation $(30,31)$ or adhesion to Col-I (32). Given the protocol described (32), the Col-I substrate used appeared to be a non-gel-form, which piqued our curiosity in studying the effect of Col-I gels on EMT induction.

Cadherins are the transmembrane components of the adherence junction at cell-cell contact sites, comprising an extracellular domain responsible for cell-cell homophilic interactions, a transmembrane domain, and a cytoplasmic tail that binds catenin (33). Catenin links cadherin to the actin cytoskeleton and functions in cellular signaling (33, 34). Cadherin molecules have multiple isoforms. E-cadherin is found in polarized epithelial cell-cell junctions widely, whereas E- and P-cadherin are found in squamous epithelial cell junctions. Notably, fibroblasts form intercellular junctions containing N-cadherin (33). The switching of cadherin from the epithelial to the mesenchymal type (i.e., cadherin switching) is an important process in EMT induction (11-13, 35, 36). Many epithelial cells begin to express mesenchymal markers during EMT process, including vimentin $(13,37,38)$ and fibronectin $(37,38)$. Cadherin switching and increased integrins are considered associated with cancer cell migration (13).

It is often assumed that cancer cells are anchorageindependent. Cancer cells are insensitive growth regulatory signals originating from adhesion to the ECM (39). However, accumulating evidence 
suggests that integrin-mediated contact of malignant cells to the ECM influences their behavior via signal transduction (40-42). Henriet et al. reported that human melanoma cells (M24met) cultured on collagen gels were growtharrested at the G1/S checkpoint and maintained high levels of p27KIP1 mRNA and protein through $\beta 1$ integrin-mediated mechanisms (42). We previously reported that adhesion to Col-I gels suppressed cell proliferation and PI3K/Akt pathway activation in three cancer cell lines (43). Akt activation is characteristically suppressed on collagen gels in these cells and keratinocytes $(9$, 43, 44).

This study investigates the EMT-related changes in A549 cells cultured on gels and non-gel forms of Col-I and on non-treated culture dishes with and without TGF- $\beta 1$. We assessed cell morphology, proliferation, single-cell migration and EMTrelated features.

\section{Results}

\section{Substrate-dependent cell morphology, cluster} formation and proliferation of A549 cells

A549 cells were cultured in four culture conditions: on non-treated culture dish (on NT), on non-gel form of Col-I coated culture dish (on Col-I-nongel), on fibrous Col-I gels coated culture dish (on Col-I-gel) and on non-treated culture dish with TGF- $\beta 1$ in the culture media (with TGF $\beta 1$ ). The cells were seeded at a single-cell density $\left(1.0 \times 10^{5}\right.$ cells / well of six-well culture plate) and cultured for 2 days. Cellular morphology was observed using phase-contrast microscopy. On NT, the cells showed typical epithelial cell clusters. On Col-Inon-gel, cell-cell interaction was weakened and the cell morphology was converted from an epithelial phenotype to a fibroblastic phenotype. The cell morphology on Col-I-gel differed from the previously mentioned two conditions. Cell spreading was suppressed, and some cells formed clusters (Fig.1A, arrows). The other cells did not form cell-cell contacts and maintained single-cell form (Fig. 1A, arrow heads). With TGF $\beta 1$, A549 cells spread very well, showing a fibroblastic-like morphology and not forming cell clusters (Fig. 1A).

Cell proliferation was estimated at the day 3 of culture. The cells continued proliferating on NT, on Col-I-non-gel, and with TGF $\beta 1$. Although cell proliferation on Col-I-gel was maintained, it was markedly suppressed compared with the other conditions (Fig. 1B).

\section{Effects of Col-I gel on the migration of A549 cells}

We monitored A549 cells in previously mentioned four culture conditions using a time-lapse observation system to examine effects of Col-I substrates on single-cell migration. The relative distance (distance between tracking start position and current position) of eight randomly selected cells in each condition was analyzed to quantitatively estimate cell motility. A549 cells on NT exhibited limited migration. Cells hardly moved from the place where they were, proliferated and formed clusters. The mean relative distance was notably the shortest among the tested conditions (Fig. 1C, Supporting Information S-1). The cells on Col-I-non-gel spread, moved well, and did not form cell clusters. The mean relative distance was the longest among the tested conditions (Fig. 1C, Supporting Information S-2). A significant difference in the relative distance between the Col-I-non-gel and the NT groups was observed. Considering the cell image on Col-I-gel at 2 days-culture, we initially hypothesized that the cells would hardly migrate and proliferate to form clusters. Contrary to expectation, on Col-I-gel, some single-cells subsequently migrated to form cell clusters and the others did not move from the place where they were. In the cells on Col-I-gel (Fig. 1C, Supporting Information S-3) and with TGF $\beta 1$ (Fig. 1C, Supporting Information S-4), the mean relative distances fell between the above two conditions and those were significantly longer than that on NT. The mean relative distance with TGF $\beta 1$ was longer than that on Col-I-gel. Furthermore, there was a significant difference between the Col- 
I-gel and the NT groups and between the TGF $\beta 1$ and the NT groups.

\section{Switching of E- to $\mathbf{N}$-cadherin was observed in A549 cells cultured on Col-I-gel as well as with TGF $\beta 1$}

E-cadherin is the transmembrane components at cell-cell contact sites in epithelial cells (33). A549 cells cultured for 2 days were immunostained with anti-E- and N- cadherin antibodies. The cells on NT expressed E-cadherin but not $\mathrm{N}$-cadherin in cellcell contact regions. On Col-I-non-gel, both E- and $\mathrm{N}$-cadherin were hardly observed in cell-cell contact regions. On Col-I-gel, E-cadherin was hardly observed, while, $\mathrm{N}$-cadherin was observed in cell-cell contact regions. Single-cells expressed both cadherins like a dot. Following with TGF $\beta 1$, the cells spread well and showed slight cell-cell contact. These regions of slight contacts did not express E-cadherin, whereas N-cadherin was occasionally observed (Fig. 2A).

Western blotting was performed to quantify the cadherin expression. Typical western blot data are presented in Figure 2B. Cadherin expression values were normalized to that of glyceraldehyde 3phosphate dehydrogenase (GAPDH). The relative expression levels of each condition were estimated based on the expression on NT. The mean value of E-cadherin expression was the highest in cells on NT, followed by on Col-I-non-gel, on Col-I-gel, and with TGF $\beta 1$ (Fig. 2C). In contrast, the mean of $\mathrm{N}$-cadherin expression was the lowest in cells on NT and increased in the order of on Col-I-non-gel, on Col-I-gel, and with TGF $\beta 1$ (Fig. 2D). Ecadherin expression levels on Col-I-gel and with TGF $\beta 1$ were significantly lower than that on NT. In the case of N-cadherin, those on Col-I-gel and with TGF $\beta 1$ were significantly higher than that on NT. Significant differences were observed in both $\mathrm{E}$ - and N- cadherin between the Col-I-gel and the NT groups and between the TGF $\beta 1$ and on NT groups (Fig.2C, 2D). The western blotting results did not coincide with the immunohistochemical observations of E-cadherin on Col-I-non-gel or Ncadherin with TGF $\beta 1$. We hypothesized this was caused by loose cell-cell contacts due to scattered cells (Fig.1A). Thus, cadherins might not accumulate and would be invisible by immunohistochemical analysis. A considerable amount of E-cadherin expression was observed by western blotting on Col-I-gel, but was not observed by immunostaining. The reason for this is unclear, it could be because of the mixture of single cells and cell clusters on Col-I-gel (Fig. 1A).

\section{Vimentin and integrins did not increase in cells on Col-I-gel, unlike those with TGF $\beta 1$}

Vimentin is a type III intermediate filament found in mesenchymal cells, maintaining cell and tissue integrity (45). Increasing vimentin expression enhances directed cell migration, and the vimentin network regulates the actin cytoskeleton, which is associated with cell migration (46). We stained cells cultured for 2 days in each condition with antivimentin antibody. Immunostaining did not show vimentin in A549 cells on NT, on Col-I-non-gel and on Col-I-gel. In contrast, vimentin was present in the spreading A549 cells with TGF 31 (Fig. 3A). Typical western blot data are presented in Figure 3B. Vimentin expression was measured and normalized to that of GAPDH. The expression levels of each condition were estimated based on that on NT. Western blottig analysis corroborated the immunohistochemical observations. A significant difference between the Col-I-gel and the TGF $\beta 1$ groups and between the TGF $\beta 1$ and the NT groups was observed (Fig. 3C).

Integrins are heterodimeric cell surface receptors that composed of $\alpha$ and $\beta$ subunits. They bind between the ECM and the inside of the cytoskeleton $(47,48)$. Integrin $\alpha 1 \beta 1, \alpha 2 \beta 1, \alpha 10 \beta 1$, and $\alpha 11 \beta 1$ are reported as collagen receptors. Most integrins have intracellular linkages to the actin cytoskeleton (47). Integrins are thought to contribute to the cancer cell properties of limitless proliferation, invasion, promotion of tumor angiogenesis, evasion of apoptosis, and the development of resistance to growth suppressors (48). Immunostaining of the cell-cell contact regions and in cell peripheral regions of A549 cells 
cultured on NT, on Col-I-non-gel, and on Col-I-gel showed integrin $\beta 1$. Actin was also observed in the peripheral regions of cells. The stained images of integrin $\beta 1$ and actin on NT, on Col-I-non-gel, and on Col-I-gel were similar. With TGF $\beta 1$, the cells spread well, and notably, actin filaments developed as long straight lines (arrows, Fig. 4A). The cellular expression of integrin $\beta 1$ and $\alpha 2$ was measured using flow cytometry. The expression ratio of integrins was standardized against the mean values of the fluorescein intensity of cells cultured on NT. In both cells cultured on Col-I-non-gel and on ColI-gel, the mean integrin $\beta 1$ and $\alpha 2$ expression values were the same as those on NT (Fig. 4B and Table 1). By contrast, the mean values of integrins $\beta 1$ and $\alpha 2$ increased by 1.13 - fold and 1.94-fold, respectively, with TGF $\beta 1$ (Fig. $4 \mathrm{C}$ and Table 1).

\section{Discussion}

In this study, we demonstrated that adhesion to Col-I fibrous gels induced a unique transition in A549 cells, i.e. E- to $\mathrm{N}$-cadherin switching, forming cell clusters via N-cadherin and upregulation of single-cell motility as compared with NT. The mean value of relative distance on Col-I-gel was shorter than that with TGF $\beta 1$ or on Col-I-non-gel. Such behaviors differed from that of with TGF $\beta 1$, as a typical EMT-induced condition. The similarities and differences of A549 cells between on Col-I-gel and with TGF $\beta 1$ are summarized in Table 2.

A few papers have been reported for EMT induction by adhesion to Col-I in A549 cells (32, 49). Shintani et al. showed that adhesion to Col-I promoted single-cell scattering. Their results were close to that of our Col-I-non-gel condition, but there were some differences (32). We think that the main cause of this is due to the different Col-I treatment protocols. Since Shintani et al. did not mention the Col-I treatment protocols in detail, the gel-forming states were unknown $(29,32,35)$. Because Col-I forms fibrils under physiological conditions, Col-I molecules coated on culture dishes may form fibrils during the cell culture period. Even if gels may not form, it is highly possible to form fibrils depends on the amount of applied Col-I. Thus, a different Col-I treatment protocol would lead to different fibril-forming states on the culture dish. However, Col-I treatment protocols are not unified in general, and the state of fibril formation on culture dishes has received little attention.

Gel culture systems utilizing Col-I fibrils are thought to be better to mimic the physiological environment than those using the non-gel form of Col-I $(50,51)$. Considering the published papers, there are at least three essential difference between fibrous Col-I gels and Col-I-non-gel form as culture substrates (52-54). First, the specific integrin-recognition sites on collagen molecules are masked on fibrils (52). Second, the matrix stiffness of gel-formed Col-I fibrils is lower than that of the plastic dish surface (53). Third, the morphology of Col-I gel surface is porous and not flat (54). Baker and coworkers have reported differences in cell morphology and proliferation on three-dimensional gel culture systems between flat hydrogel (e.g., polyacrylamide gels) and synthetic fibrous materials (e.g., using electrospinning) (54). Each experimental system in these papers (52-54) is designed to focus on a single factor, but these factors can collectively affect cells in Col-I gel culture system. We think it is crucial to study the phenomena in Col-I gel culture system to elucidate comprehensive roles of Col-I in the living body. For example, increasing matrix stiffness induces EMT $(55,56)$. In contrast, we observed that the switching from E- to N-cadherin was more prominent on softer Col-I-gel than that on harder Col-I-non-gel (Fig. 2), suggesting that the effect of matrix stiffness is important but not an only determinant factor to induce cadherin switching. Shukla et al. reported EMT induction and cell migration in A549 cells focusing on the effects of Col-I substrate stiffness. They examined cell migration parameters and cadherin switching on collagen-coated polydimethylsiloxane gels with varying degrees of stiffness. The migration parameters depended on substrate stiffness, while E- to N-cadherin switching was unaffected (49). 
A correlation between $\mathrm{N}$-cadherin expression and cell motility is now under debate. $\mathrm{N}$-cadherin expression is upregulated in some invasive cancer cell lines $(57,58)$. N-cadherin overexpression induces an invasive morphology in squamous tumor cells (59) and breast cancer cells (60) in vitro. $\mathrm{N}$-cadherin knockdown cells exhibit reduced migration and a limited ability to scatter on collagen in pancreatic cancer cells, and N-cadherin expression promote metastasis in an orthotopic model (29). However, there is no correlation between $\mathrm{N}$-cadherin expression and invasion in astrocytoma and glioblastoma $(61,62)$. In our study, there was no simple correlation between Ncadherin expression (Fig. 2D) and cell migration (Fig. 1C). We think this is caused by the balance of the following two factors. Signal activation via adhesion to cell recognition sites on Col-I fibrils promotes cadherin switching induction and migration in cells. On the other hand, gel natures of Col-I, e. g., gel stiffness and an uneven surface condition might suppress cell migration. Considering each factor, more study is necessary to elucidate the correlation between cadherin switching and cell motility on fibrous materials like Col-I gels.

In EMT induction by TGF- $\beta$ signaling activation, EMT-related signals often lead and enhance TGF$\beta$ autocrine. TGF- $\beta$ autocrine sometimes goes into a closed-loop system (autocrine loop), and it contributes to enhance and maintain the mesenchymal state in cells $(25,63,64)$. Once TGF$\beta$ signaling activates, TGF- $\beta$ autocrine activation maintains via $\mathrm{TGF}-\beta / \mathrm{ZEB} / \mathrm{miR}-200$ signaling network to keep the mesenchymal phenotype in MDCK cells (63). Sphingosine-1-phosphateinduced EMT is mediated by syndecan- 1 activation and TGF- $\beta$ autocrine (64). In our study, the expression of mesenchymal cytoskeleton (vimentin) and collagen receptor (integrin $\alpha 2 \beta 1$ ) was increased in TGF- $\beta 1$-treated cells. In contrast, they did not increase on both states of Col-I (Fig. 3, 4). Thus, we considered that TGF- $\beta$ signal activation did not affect cells on Col-I. The development of the cytoskeleton and integrinECM interaction enhances cell migration (65).
The interstitial Col-I fibril lattices function as both a cell scaffold and a barrier to prevent cell migration in cancer invasion process $(6,66)$. Furthermore, EMT induction via adhesion to Col-I is thought to enhance cancer invasion (32). The effects of adhesion to Col-I (regardless of the state of fibril formation) are still ambiguous. Ncadherin-mediated cell cluster formation in cadherin switching induced-cells is a unique phenomenon. It may provide the key to understand as yet unelucidated biological and pathological phenomena. A schematic view of the roles of ColI fibrils in cancer invasion is shown in Figure 5.

Many individuals may have undiagnosed tumors in their bodies for several years $(67,68)$. Bissell and Hines reported that the microenvironment surrounding the tumors was the source of tumorsuppressive signals in these cases (68). Thus, Col-I fibrils may be one of the niche factors affecting cancer dormancy.

\section{Experimental procedures}

\section{Cell culture}

Human lung cancer cell line A549 cells were provided by the RIKEN BRC through the National Bio-Resource Project of the Next Japan. The cells were maintained in Dulbecco's modified Eagle Medium (DMEM; Sigma-Aldrich, St. Louis, MO, USA) supplemented with $10 \%$ fetal bovine serum (FBS; Biowest, Nuaille, France).

\section{TGF- $\beta 1$ treatment}

A549 cells were treated with $2 \mathrm{ng} / \mathrm{ml}$ TGF- $\beta 1$ (Sigma-Aldrich, Merck KGaA, Darmstadt, Germany) for 2 days in DMEM with $10 \%$ FBS on non-treated culture dish surfaces (31) to induce EMT.

\section{Preparation of culture substrates}

Acid-soluble bovine Col-I was obtained from Nippi Inc. (Tokyo, Japan). The plastic surfaces of tissue culture plates were treated with Col-I in two 
ways as previously described (9). For the non-gel form, the wells were coated with Col-I $(10 \mu \mathrm{g} / \mathrm{ml})$ in $1 \mathrm{mM} \mathrm{HCl}$ for $1 \mathrm{~h}$ at room temperature. For the gel form, $1 \mathrm{mg} / \mathrm{ml}$ Col-I solution in PBS (-) (phosphate-buffered saline without calcium and magnesium) was added to the wells of a culture plate (96-well plate, $0.1 \mathrm{ml} /$ well; six-well plate, 1 $\mathrm{ml} /$ well) and incubated for $1 \mathrm{~h}$ at $37{ }^{\circ} \mathrm{C}$ in a humidified $\mathrm{CO}_{2}$ incubator $(9,43)$.

\section{Analysis of cell proliferation}

The number of living cells was estimated using a WST-8 (modified tetrazolium salt) cell proliferation kit (Cell Counting Kit-8; Dojin, Kumamoto, Japan) according to the manufacturer's protocol. Briefly, A549 cells $\left(5.0 \times 10^{3}\right.$ cells/well $)$ were cultured using DMEM including $10 \%$ FBS in a 96-well tissue culture plate treated with or without Col-I. The absorbance was measured at $450 \mathrm{~nm}$ using SH-9000 Lab microplate reader (Corona Electric Co., Ltd., Ibaraki, Japan). Each condition was assessed in triplicate and the results were expressed as means \pm standard deviation (SD).

\section{Analysis of cell migration using time-lapse microscopy}

The migration of A549 cells was monitored using the time-lapse function of BZ-X800 microscope system (Keyence, Osaka, Japan) housed in a $37^{\circ} \mathrm{C}$ temperature-controlled chamber. Culture plates (six-well) were used in the non-treated condition or preincubated with Col-I (gels or non-gel-form). The cells were seeded at a concentration of $1.0 \times$ $10^{5}$ cells/well on the surface of both forms of Col-I and on non-treated dish surfaces with or without TGF- $\beta 1(2 \mathrm{ng} / \mathrm{ml})$ and preincubated in a $\mathrm{CO}_{2}$ incubator for $2 \mathrm{~h}$. Subsequently, the cells were placed in a microscope chamber and monitored for $48 \mathrm{~h}$. The relative distance (distance between the tracking start position and the current position) of eight randomly selected cells in each condition was assessed using the BZ-H4K Motion Analysis Application (Keyence, Japan), expressed as means \pm SD. Calculations were performed using Microsoft Excel for Mac (ver. 16.16.24).
Differences between two individual groups were analyzed using Student's t-test (two-tailed). Results were considered significantly different when $P<0.05$.

\section{Antibodies}

Mouse monoclonal E-cadherin antibody (HECD-1) was purchased from Takara Bio Inc. (Shiga, Japan). Rabbit monoclonal N-cadherin (D4R1H), vimentin (D21H3), and GAPDH (14C10) antibodies were purchased from Cell Signaling Technology, Inc. (Beverly, MA, USA). Mouse monoclonal integrin $\beta 1$-activating (TS2 /16) and mouse monoclonal integrin $\alpha 2$ (P4B4) antibodies were purchased from Santa Cruz Biotechnology, Inc. (Dallas, TX, USA). Peroxidase-conjugated secondary antibodies against rabbit IgG and mouse IgG were purchased from Cell Signaling Technology, Inc. The Alexa Fluor 488-conjugated antibodies against mouse IgG and rabbit IgG were obtained from Santa Cruz Biotechnology, Inc. Phalloidintetramethylrhodamine B isothiocyanate was obtained from Sigma-Aldrich.

\section{Immunohistochemical analysis}

Prior to cell culture, twelve-well Teflon-coated slides (Thermo Fisher Scientific, Waltham, MA, USA) were treated with Col-I as previously described (9). Following the growth of cells on the slides for 2 days on each condition, they were washed with PBS (-) and fixed with 4\% paraformaldehyde for $1 \mathrm{~h}$ at room temperature. Then, the cells were washed with PBS (-), permeabilized, incubated in 1\% FBS/PBS (-) for 1 $\mathrm{h}$ at room temperature, and incubated with monoclonal antibodies overnight at $4{ }^{\circ} \mathrm{C}$. The cells were washed thrice with PBS-Tween 20 and incubated with Alexa Fluor 488-conjugated secondary antibody (diluted 1: 200 in PBS (-)) for $1 \mathrm{~h}$. Finally, the cells were stained with VECTASHIELD Antifade Mounting Medium with DAPI (Vector Laboratories, Burlingame, CA, USA) and observed using an FV1200 laser scanning microscope (Olympus, Tokyo, Japan). 


\section{Western blotting}

Prior to the preparation of samples for western blot analysis, A549 cells $\left(1.0 \times 10^{5}\right.$ cells/well $)$ in sixwell tissue culture plates containing $1 \mathrm{ml}$ DMEM (10) were cultured for 2 days on each condition. In the case of Col-I-gel, the cells and gels were gathered together and centrifuged. After discarding the supernatant, the pellet was washed with PBS $(-)$ and incubated on ice for $5 \mathrm{~min}$ in $0.1 \mathrm{ml}$ cell lysis buffer (Cell Signaling Technology, Inc.). In the other conditions, the cells were washed with PBS $(-)$ and lysed on ice for $5 \mathrm{~min}$ in $0.05 \mathrm{ml}$ cell lysis buffer as previously described (9). The samples were electrophoresed on a $10 \%$ polyacrylamide gel and electrophoretically transferred to nitrocellulose membranes. The membranes were blocked overnight at $4{ }^{\circ} \mathrm{C}$ with $2 \%$ nonfat dry milk in trisbuffered saline (TBS) containing $0.05 \%$ Tween-20 (TBS-T), followed by incubation with primary antibody diluted in TBS-T. The bands were visualized using Amersham ECL Prime Western Blotting Detection Reagents (GE Healthcare, Amersham, UK) according to the manufacturer's instructions. Four independent experiments were conducted. The protein concentrations were determined using Fiji software (Media Cybernetics, Rockville, MD), and expressed as means \pm SD. Calculations were performed using Microsoft Excel for Mac (ver. 16.16.24). Differences between two individual groups were analyzed using Student's t-test (two-tailed). Results were considered significantly different when $P<0.05$.

\section{Flow cytometry}

Prior to the flow cytometric analysis, A549 cells $\left(1.0 \times 10^{5}\right.$ cells/well $)$ were seeded in six-well tissue culture plates and cultured on each condition for 2 days. On Col-I-gel, the cells were gathered from Col-I gels using $1 \mathrm{mg} / \mathrm{ml}$ collagenase (Wako, Japan) at $37{ }^{\circ} \mathrm{C}$ for $5 \mathrm{~min}$ and then centrifuged and discarded supernatant. Precipitated cells were separated into single cells by treatment with $0.25 \%$ trypsin- $0.02 \%$ (trypsin-EDTA) (Novozymes North America, Inc., Franklinton, NC, USA) at $37{ }^{\circ} \mathrm{C}$ for $5 \mathrm{~min}$. In the other conditions, the cells were treated with $1 \mathrm{mg} / \mathrm{ml}$ collagenase (Wako) at $37^{\circ} \mathrm{C}$ for 5 min and gathered with trypsin-EDTA treatment at $37^{\circ} \mathrm{C}$ for $5 \mathrm{~min}$. Then the cells were resuspended in the treatment buffer and stained with anti-bodies as previously described (69). Immunofluorescentstained cells were analyzed using a FACSAria II Flow Cytometer (BD Bioscience, Franklin Lakes, NJ, USA) and the resultant data were analyzed using the FlowJo software (Tomy Digital Biology Co., Ltd., Tokyo, Japan). More than 5,000 cells were examined for each condition. 


\section{Data availability}

The datasets available from the corresponding author on request.

\section{Acknowledgement}

This work was performed in part under the Cooperative Research Program of Institute for Protein

Research, Osaka University (CR-15- 01-7).

The authors would like to thank Enago (www.enago.jp) for the English language review.

\section{Conflict of interest}

There is no conflict of interest for all authors.

\section{References}

1. Grinnell, F. (1982) Cell-collagen interactions: Overview. Methods Enzymol 82, 499-503

2. Kleinman, H. K., Kibbery, M. C., Cannon, F. B., Weeks, B. S., Grant, D. S. (1995) Use of extracellular matrix and its components in culture. In "Extracellular Matrix: A Practical Approach" (M. A. Haralson and J. R. Hassell, Eds.), 1st ed., IRL Press, Oxford: 289-301

3. Kleinman, H. K. (1982) Fibroblast adhesion to collagen substrates. Methods Enzymol 82, 503-508

4. Suzuki, Y., Someki, I., Adachi, E., Irie, S., Hattori, S. (1999). Interaction of collagen molecules from the aspect of fibril formation: Acid-soluble, alkali-treated and MMP1-digested fragments of type I collagen. J Biochem 126, 54-67

5. Latifi, N., Asgari, M., Vail, H., Mogeau, L. (2018) A tissue-mimetic nano-fibrillar hybrid injectable hydrogel for potential soft tissue engineering applications. Scientific Rep doi:101038/s41598-01718523-3

6. Dettman, R. W., Simon, H.-G. (2018) Rebooting the collagen gel: Artificial hydrogels for the study of epithelial mesenchymal transition. Develo Dynamics 247, 332-339

7. Nishiyama, T., Tominaga, N., Nakajima, K., Hayashi, T. (1988) Quantitative evaluation of the factors affecting the process of fibroblast-mediated collagen gel contraction by separating the process into three phases. Coll Relat Res 8, 259-273

8. Koyama, H., Raines, E. W., Bornfeldt, K. E., Roberts, J. M., Ross, R. (1996) Fibrillar collagen inhibits arterial smooth muscle proliferation through regulation of Cdk2 inhibitors. Cell 87, 10691078

9. Fujisaki, H., Hattori, S. (2002) Keratinocyte Apoptosis on Type I collagen gel caused by lack of laminin 5/10/11 deposition and Akt signaling. Exp Cell Res 280, 255-269 
10. Liu, X., Long, X., Liu, W., Yao, G., Zhao, Y., Hayashi, T., Hattori, S., Fujisaki, H., Ogura, T., Tashiro, S. I., Onodera, S., Yamato, M., Ikejima, T. (2018) Differential levels of reactive oxygen species in murine preadipocyte 3T3-L1 cells cultured on type I collagen molecule-coated and gelcovered dishes exert opposite effects on NF-kB-mediated proliferation and migration. Free Radic Res 52, 913-928

11. Thiery, J. P., Acloque, H., Huang, R. Y. J., Nieto, A. (2009) Epithelial -Mesenchymal Transition on Development and Disease. Cell 139, 871-890

12. Yang, J., Antin, P., Berx, G., Blanpain, C., Brabletz, T., Bronner, M., Campbell, K., Cano, A., Casanova, J., Christofori, G., Dedhar, S., Derynck, R., Ford, H. L., Fuxe, J., de Herreros, A. G., Goodall, G. J., Hadjantonakis, A.-K., Huang, R. J. Y., Kalcheim, C., Kalluri, R., Kang, Y., KhewGoodall, Y., Levine, H., Liu, J., Longmore, G. D., Mani, S. A, Massague, J., Mayor, R., McClay, D., Mostov, K. E., Newgreen, D. F., Nieto, M. A., Puisieux, A., Runyan, R., Savagner, P., Stranger, B., Stemmler, M. P., Takahashi, Y., Takeichi, M., Theveneau, E., Thiery, J. P., Thompson, E. W., Weinberg, R. A., Williams, E. D., Xing, J., Zhou, B. P., Sheng, G., TEMTIA (2020) Guidelines and definitions for research on epithelial-mesenchymal transition. Nat Rev Mol Cell Biol doi: 10.1038/s41580-020-0237-9

13. Hugo, H., Ackland, M. L., Blick, T., Lawrence, M. G., Clements, J. A., Williams, E. D., Thompson, E. W. (2007) Epithelial-mesenchymal and mesenchymal-epithelial transitions in carcinoma progression. J Cell Physiol 213, 374-383

14. Chaffer, C. L., Brennan, J. P., Slavin, J. L., Blick, T., Thompson, E. W., Williams, E. D. (2006) Mesenchymal-to-Epithelial Transition Facilitates Bladder Cancer Metastasis: Role of Fibroblast Growth Factor Receptor-2. Cancer Res 66, 11271-11278

15. Fitzgerald, M. P., Gourronc, F., Teoh, M. L., Provenzano, M. J., Case, A. J., Martin, J. A., Domann, F. E. (2011) Human Chondrosarcoma Cells Acquire an Epithelial-Like Gene Expression Pattern via an Epigenetic Switch: Evidence for Mesenchymal-Epithelial Transition During Sarcoma genesis. Sarcoma, doi:10.1155/2011/598218

16. Greenburg, G., Hay, E. D. (1982) Epithelia suspended in collagen gels can lose polarity and express characteristics of migrating mesenchymal cells. J Cell Biol 95, 333-339

17. Blanco, M. J., Moreno-Bueno, G., Sarrio, D., Locascio, A., Cano, A., Palacios, J., Nieto, M. A. (2002) Correlation of Snail expression with histological grade and lymph node status in breast carcinomas. Oncogene 21, 3241-3246

18. Thuault, S., Tan, E-J., Peinado, H., Cano, A., Heldin, C. H., Moustakas, A. (2008) HMGA2 and Smads co-regulate SNAIL 1 expression during induction of epithelial-to-mesenchymal transition. $J$ Biol Chem 283, 33437-33446

19. Terry, S., Savagner, P., Ortiz-Cuaran, S., Mahjoubi, L., Saintigny, P., Thiery, J. P., Chouaib, S. (2017) New insights into the role of EMT in tumor immune escape. Mol Oncol 11, 824-846

20. Thiery, J. P. (2002) Epithelial-mesenchymal transitions in tumour progression. Nature Rev Cancer 2, 442-454

21. Cufí, S., Vazquez-Martin, A., Oliveras-Ferraros, C., Martin-Castillo, B., Joven, J. (2010) Metformin against TGF- $\beta$-induced epithelial-to mesenchymal transition (EMT): from cancer stem cells to aging associated fibrosis. Cell Cycle doi: 10.4161/cc.9.22.14048

22. Moustakas, A., Heldin, C. H. (2007) Signaling networks guiding epithelial mesenchymal transitions during embryogenesis and cancer progression. Cancer Sci 98, 1512-1520

23. Lee, J.M., Dedhar, S., Kalluri, R., Thompson, E. W. (2006) The epithelial-mesenchymal transition: new insights in signaling, development, and disease. J Cell Biol 172, 973-981

24. Roberts, A. B., McCune, B. K., Spom, M. B. (1992) TGF-beta: regulation of extracellular matrix. Kidney Int 41, 557-559

25. Larocco, C., Cohen, J. R., Fernando, R. I., Huang, B., Hamilton, D. H., Palena, C. (2013) An 
autocrine loop between TGF- $\beta 1$ and the transcription factor Brachyury controls the transition of human carcinoma cells into a mesenchymalphenotype. Mol Cancer Ther 12, doi:10.1158/15357163. MCT-12-1007

26. Sander, E. E., van Delft, S., ten Klooster, J. P., Reid, T., van der Kammen, R. A., Michiels, F., Collard, J. G. (1998) Matrix-dependent Tiam1/Rac signaling in epithelial cells promotes either cell-cell adhesion or cell migration and is regulated by phosphatidylinositol 3-kinase. J Cell Biol 143, 1385-1398

27. Huang, H., Svoboda, R. A., Lazenby, A. J., Saowapa, J., Chaika, N., Ding, K., Wheelock, M. J., Johnson, K. (2016) Up-regulation of N-cadherin by collagen I -activated discoidin domain receptor 1 in pancreatic cancer requires the adaptor molecule Shc1. J Biol Chem 291, 23208-23223

28. Aguilera, K. Y., Rivera, L. B., Hur, H., Carbon, J. G., Toombs, J. E., Goldstein, C. D., Dellinger, M. T., Castrillon, D. H., Brekken, R. A. (2014) Collagen signaling enhances tumor progression after anti-VEGF therapy in a murine model of pancreatic ductal adenocarcinoma. Cancer Res $\mathbf{7 4 ,}$ 1032-1044

29. Shintani, Y., Hollingsworth, M. A., Wheelock, M. J., Johnson, K. R. (2006) Collagen I promotes metastasis in pancreatic cancer by activating c-Jun NH 2 -terminal kinase 1 and up-regulating NCadherin expression. Cancer Res 66, 11745-11753

30. Tirino, V., Camerlingo, R., Bifulco, K., Irollo, E., Montella, R., Paino, F., Sessa, G., Carriero, M. V., Normanno, N., Rocco, G., Pirozzi, G. (2013) TGF- $\beta 1$ exposure induces epithelial to mesenchymal transition both in CSCs and non-CSCs of the A549 cell line, leading to an increase of migration ability in the CD133+ A549 cell fraction. Cell Death Dis 4, e620

31. Yu, J. I., Dou, Y., Zhao, Q., Zhang, J., Yang, Y., Wang, T., Xia, Y., Dai, Y., Wei, Z. (2016) Paeoniflorin suppresses TGF- $\beta$ mediated epithelial- mesenchymal transition in pulmonary fibrosis through a Smad-dependent pathway. Acta Pharmacol Sin 37, 794-804

32. Shintani, Y., Maeda, M., Chaika, N., Johnson, K. R., Wheelock, M. J. (2008) Collagen I Promotes EMT in Lung Cancer Cells via TGF-beta3 Signaling. Am. J. Respir. Cell Mol Biol 38, 95-104

33. Wheelock, M. J., Johnson, K. R. (2003) Cadherin-mediated cellular signaling. Curr Opin Cell Biol 15, 509-514

34. Angst, B. D., Marcozzi, C., Magee, A. I. (2001) The cadherin superfamily: diversity in form and function. J Cell Sci 114 (Pt 4), 629-641

35. Wheelock, M. J., Shintani, Y., Maeda, M., Fukumoto, Y., Johnson, K. R. (2008) Cadherin switching. J Cell Sci 121, 727-735

36. Thiery, J. P., Sleeman, J. P. (2006) Complex networks orchestrate epithelial- mesenchymal transitions. Nat Rev Mol Cell Biol 7, 131-142

37. Chen, X. F., Zhang, H. J., Wang, H. B., Zhu, J., Zhou, W. Y., Zhang, H., Zhao, M. C., Su, J. M., Gao, W., Zhang, L., Fei, K., Zhang, H. T., Wang, H. Y. (2012) Transforming growth factor- $\beta 1$ induces epithelial-to-mesenchymal transition in human lung cancer cells via PI3K/Akt and MEK/Erk1/2 signaling pathways. Mol Biol Rep 39, 3549-3556

38. Rout-Pitt, N., Farrow, N., Parsons, D., Donnelley, M. (2018) Epithelial mesenchymal transition (EMT): a universal process in lung diseases with implications for cystic fibrosis pathophysiology. Respiratory Res 19, 136-145

39. Paoli, P., Giannoni, E., Chiarugi, P. (2013) Anoikis molecular pathway its role in cancer progression. Biochim Biophys Acta 1833, 3481-3498

40. Ben-Ze'ev, A. (1997) Cytoskeletal and adhesion proteins as tumor suppressors. Curr Opin Cell Biol 9, 99-108

41. Varner, J. A., Cheresh, D. A. (1996) Integrins and cancer. Curr Opin Cell Biol 8, 724-730

42. Henriet, P., Zhong, Z. D., Brooks, P. C., Weinberg, K. I., DeClerck, Y. A. (2000) Contact with fibrillar collagen inhibits melanoma cell proliferation by up-regulating p27KIP1. Proc Natl Acad 
Sci USA 97, 10026-10031

43. Sasaki, J., Fujisaki, H., Adachi, E., Irie, S., Hattori, S. (2011) Delay of cell cycle progression and induction of death of cancer cells on type I collagen fibrils. Connect Tissue Res 52,167-177

44. Fujisaki, H., Adachi, E., Hattori, S. (2008) Keratinocyte differentiation and proliferation are regulated by adhesion to the three-dimensional meshwork structure of type IV collagen. Connect Tissue Res 49, 426-436

45. Coulombe, P. A., Wong, P. (2004) Cytoplasmic intermediate filaments revealed as dynamic and multipurpose scaffolds. Nat Cell Biol 6, 699-706

46. Liu, C. Y., Lin, H. H., Tang, M. J., Wang, Y. K. (2015) Vimentin contributes to epithelialmesenchymal transition cancer cell mechanics by mediating cytoskeletal organization and focal adhesion maturation. Oncotarget 6, 15966-15983

47. Barczyk, M., Carracedo, S., Gullberg, D. (2010) Integrins. Cell Tiss Res 339, 269-280

48. Bianconi, D., Unseld, M., Prager, G. W. (2016) Integrins in the Spotlight of Cancer. Int J Mol Sci 17, doi:10.3390/ijms17122037

49. Shukla, V. C., Higuita-Castro, N., Nana-Sinkam, P., Ghadiali, S. N. (2016) Substrate stiffness modulates lung cancer cell migration but not epithelial to mesenchymal transition. J Biomad Material Res 104, 1182-1193

50. Kanta, J. (2015) Collagen matrix as a tool in studying fibroblastic cell behavior. Cell Adh Migr 9, 308-316

51. Gudjonsson, T., Ronnov-Jessen, L., Villadsen R., Bissell, M. J., Petersen O. W. (2003) To create the correct microenvironment: three-dimensional heterotypic collagen assays for human breast epithelial morphogenesis and neoplasia. Methods 30, 247-255

52. Zeltz, C., Orgel, J., Gullberg, D. (2014) Molecular composition and function integrin-based collagen glues- Introducing COLINBIs. Biochim Biophys Acta 1840, 2533-2548

53. Kolahi, K. S., Donjacour, A., Liu, X., Lin, W., Simbulan, R. K., Bloisel, E., Maltepe, E., Rinaudo, P. (2012) Effect of Substrate Stiffness on Early Mouse Embryo Development. Plos One 7, e41717

54. Baker, B. M., Trappmann, B., Wang, W. Y., Sakar, M. S., Kim, I. L., Shenoy, V. B., Burdick, J. A., Chen, C. S. (2015) Cell-mediated fiber recruitment drives extracellular matrix mechanosensing in engineered fibrillar microenvironments. Nat Mater 14, 1262-1268

55. Wei, S. C., Yang, J. (2016) Forcing through tumor metastasis: the interplay between tissue rigidity and epithelial-mesenchymal transition. Trends Cell Biol 26, 111-120

56. Rice, A. J., Cortes, E., Lachowski, D., Cheung, B. C. H., Karim, S. A., Morton, J. P., Hernandez, A. R. (2017) Matrix stiffness induces epithelial-mesenchymal transition and promotes chemoresistance in pancreatic cancer cells. Oncogenesis 6, e352; doi 10.1038/oncsis.2017.54

57. Hazan, R. B., Kang, L., Whooley, B. P., Borgen, P. I. (1997) N-cadherin promotes adhesion between invasive breast cancer cells and the stroma. Cell Adhes Commun 4, 399-411

58. Tran, N. L., Nagle, R. B., Cress, A. E., Heimark, R. L. (1999) N-Cadherin expression in human prostate carcinoma cell lines. An epithelial-mesenchymal transformation mediating adhesion with Stromal cells. Am J Pathol 155, 787-798

59. Islam, S., Carey, T. E., Wolf, G. T., Wheelock, M. J., Johnson, K. R. (1996) Expression of Ncadherin by human squamous carcinoma cells induces a scattered fibroblastic phenotype with disrupted cell-cell adhesion. J Cell Biol 135, 1643-1654

60. Nieman, M. T., Prudoff, R. S., Johnson, K. R., Wheelock, M. J. (1999) N-cadherin promotes motility in human breast cancer cells regardless of their E-cadherin expression. J Cell Biol 147, 631-644

61. Shinoura, N., Paradies, N. E., Warnick, R. E., Chen, H., Larson, J. J., Tew, J. J. (1995) Expression of N-cadherin and alpha-catenin in astrocytomas and glioblastomas. Br J Cancer 72, 627-633

62. Perego, C., Vanoni, C., Massari, S., Raimondi, A., Pola, S., Cattaneo, M. G. (2002) Invasive 
behavior of glioblastoma cell lines is associated with altered organization of the cadherin-catenin adhesion system. J Cell Sci 115, 3331-3340

63. Gregory, P. A., Bracken, C. P., Smith, E., Bert, A. G., Wright, J. A., Roslan, S., Morris, M., Wyatt, L., Farshid, G., Lim, Y-Y., Lindeman, G. J., Shannon, M. F., Drew, P. A., Khew-Goodall, Y., Goodall, G. J. (2011) An autocrine TGF- $\beta / Z E B / m i R-200$ signaling network regulates establishment and maintenance of epithelial-mesenchymal transition. Mol Biol Cell 22, 1686-1698

64. Zeng, Y., Yao, X., Chen, L., Yan, Z., Liu, J., Zhang, Y., Feng, T., Wu, J., Liu, X. (2016) Sphingosine-1-phosphate induced epithelial-mesenchymal transition of hepatocellular carcinoma via an MMP-7/ syndecan-1/ TGF- $\beta$ autocrine loop. Oncotarget 7, 63324-63337

65. Nakatsuji, Y., Nishio, Y., Tani, N., Adachi, K., Ohmichi, M., Hisamoto, K., Morishige, K., Kurachi, H., Tasaka, K., Murata, Y., Matsuura, N. (2003) Epidermal growth factor enhances invasive activity of BeWo Choriocarcinoma cells by including $\alpha 2$ integrin expression. Endocrine J 50, 703714

66. van Helvert, S., Storm, C., Friedl, P. (2018) Mechanoreciprocity in cell migration. Nat Cell Biol 20, $8-20$

67. Folkman, J., Kalluri, R. (2004) Cancer without disease. Nature 427, 787

68. Bissell, M. J., Hines, W. C. (2011) Why don't we get more cancer? A proposed role of the microenvironment in restraining cancer progression. Nat Med 17, 320-329

69. Fujisaki, H., Futaki, S., Yamada, M., Sekiguchi, K., Hayashi, T., Ikejima, T., Hattori, S. (2018)

Respective optimal calcium concentrations for proliferation on type I collagen fibrils in two keratinocyte line cells, HaCaT and FEPE1L-8. Regenerative Therapy 8, 73-79

\section{Footnotes}

The abbreviations:

Col-I

Col-I-gel

Col-I-non-gel

DMEM

DMEM (10)

ECM

EMP

EMT

FBS

GAPDH

NT

PBS (-)

PBS-T

PI3K type I collagen

fibrous Col-I gels

non-gel form of Col-I

Dulbecco's Modified Eagle Medium

DMEM supplemented with $10 \%$ fetal bovine serum

extracellular matrix

Epithelial-mesenchymal plasticity

Epithelial-to-mesenchymal transition

fetal bovine serum

glyceraldehyde 3-phosphate dehydrogenase

non-treated cell-culture dish

phosphate-buffered saline without calcium and magnesium

PBS (-) containing $0.05 \%$ Tween 20

phosphatidylinositol 3-kinase 
ROS reactive oxygen species

TBS tris-buffered saline

TBS-T TBS containing $0.05 \%$ Tween- 20

TGF- $\beta \quad$ transforming growth factor- $\beta$

TGF $\beta 1 \quad$ non-treated culture dish with TGF- $\beta 1$ in the culture media

Table 1. Comparative integrin expression ratio in each culture condition comparing with NT

\begin{tabular}{|c|c|c|}
\hline Culture condition & $\begin{array}{c}\text { Integrin } \beta 1 \text { expression ratio } \\
\text { (folds) }\end{array}$ & $\begin{array}{c}\text { Integrin } \alpha 2 \text { expression ratio } \\
\text { (folds) }\end{array}$ \\
\hline Col-I-non-gel & 1.02 & 0.94 \\
\hline Col-I-gel & 0.87 & 1.04 \\
\hline TGF $\beta 1$ & 1.13 & 1.94 \\
\hline
\end{tabular}


Table 2. Comparative cell features between on Col-I-gel and with TGF $\beta 1$

The features of A549 cells cultured on Col-I-gel and with TGF $\beta 1$ are summarized.

Culture conditions: A549 cells cultured for 2 days on Col-I gels (Col-I-gel), on non-treated culture dish with TGF- $\beta 1$ (with TGF $\beta 1$ ).

\begin{tabular}{|l|l|}
\hline The similar features & The different features \\
\hline Cell motility up-regulation & Cell morphology \\
\hline N-cadherin up-regulation & N-cadherin localization \\
\hline E-cadherin down-regulation & Vimentin expression \\
\hline & Integrin $\alpha 2 \beta 1$ expression \\
\hline & Actin polymerization \\
\hline
\end{tabular}




\section{Figures}

A
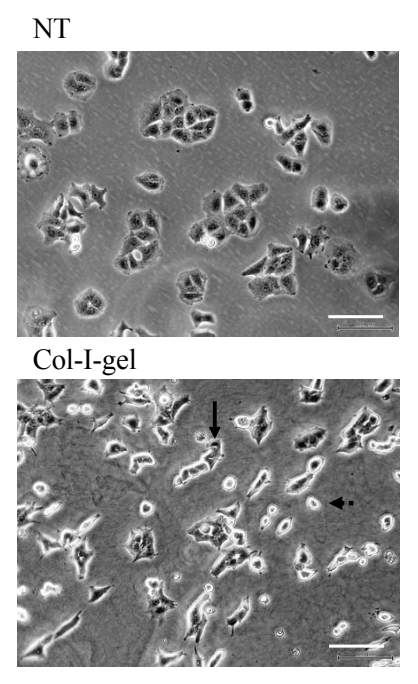

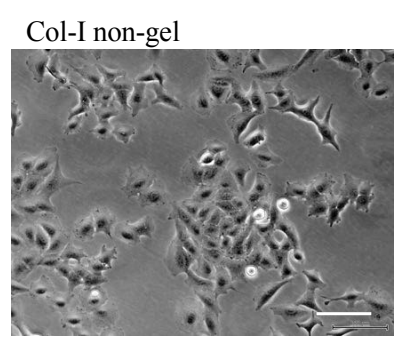

TGF $\beta 1$

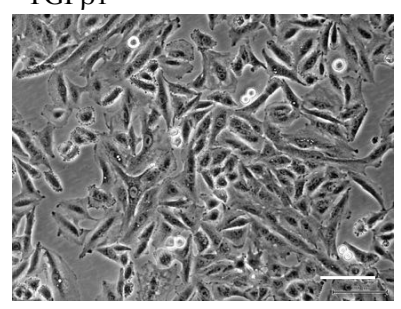

B

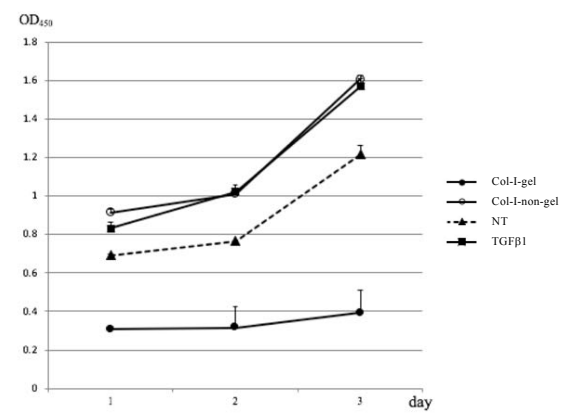

C

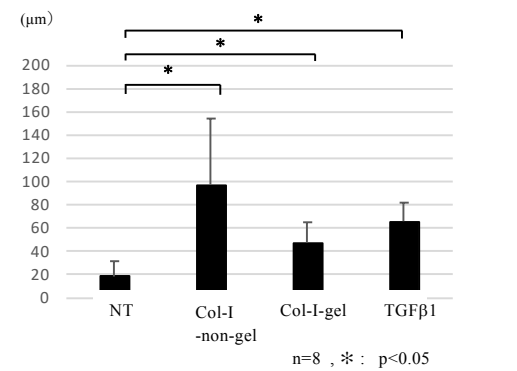

Figure 1. Morphology, proliferation and migration of A549 cells. A. A549 cells were cultured on NT, on Col-I-non-gel, on Col-I-gel and with TGF $\beta 1$ for 2 days. Cell morphology was observed using phasecontrast microscopy. Scale bars :100 $\mu \mathrm{m}$ B. The number of viable cells was estimated using the WST-8 method on days 1, 2, and 3. A549 cells were cultured on NT (dotted line, triangles), Col-I-non-gel (solid line, open circles), Col-I-gel (solid line, closed circles), and TGF $\beta 1$ (dotted line, crosses). The experiments were performed in triplicate, and data are shown as means $\pm \mathrm{SD}$. C. Monitoring of the migration of single cells for 2 days by using time-lapse microscopy. The relative distance of eight randomly selected cells on each condition was assessed. The mean relative distances are shown as means \pm SD. Differences between two individual groups were analyzed using Student's t-test. ${ }^{*} P<0.05$ 


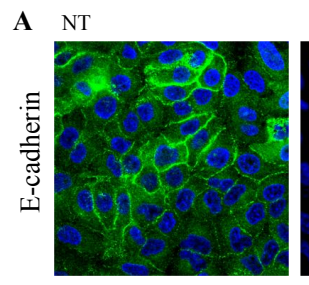

Col-I-non-gel

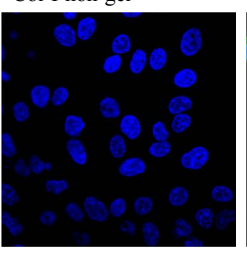

Col-I-gel

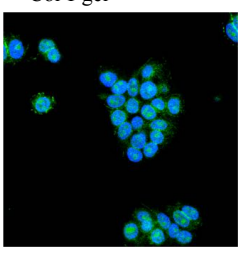

TGF $\beta 1$
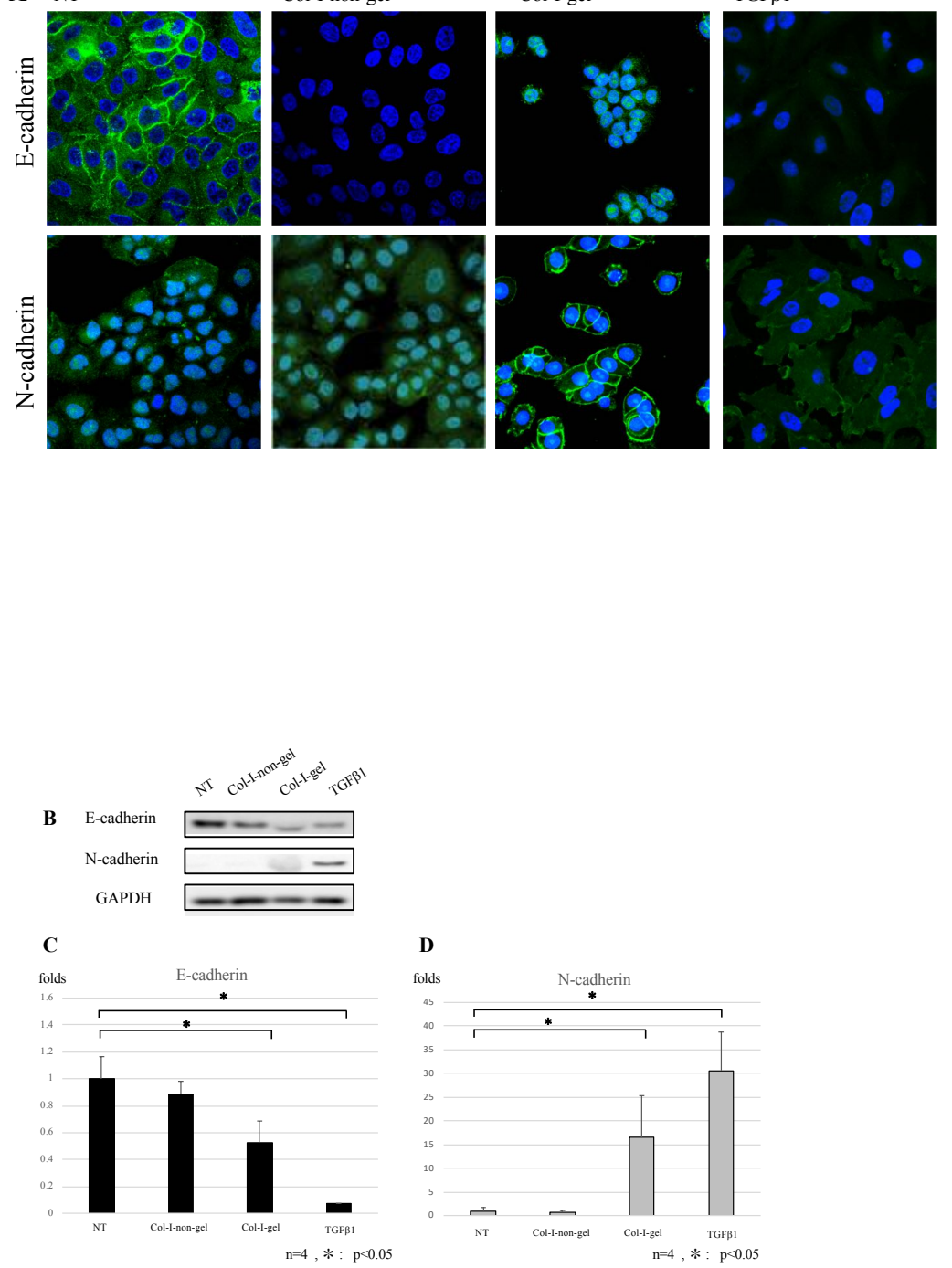

Figure 2. Expression of E-cadherin and N-cadherin in A549 cells. A. A549 cells were cultured on NT, on Col-I-non-gel, on Col-I-gel and with TGF $\beta 1$ for 2 days. Cells were stained with E- and N-cadherin antibodies and observed using a laser scanning microscope. B. Typical western blot data are shown. C. Ecadherin protein expression levels were determined from the western blots. D. N-cadherin protein expression levels were determined from the western blots. The mean values in each condition were divided by that of NT. Differences between two individual groups were analyzed using Student's t-test. $* P<0.05$ 
A NT

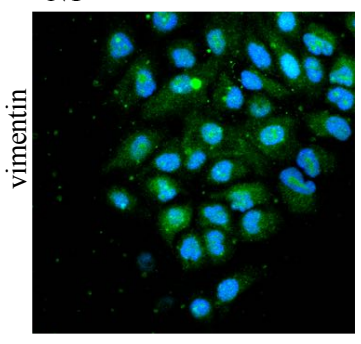

Col-I-non-gel

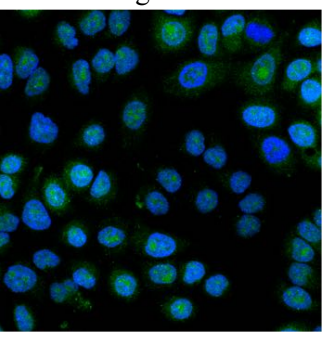

Col-I-gel

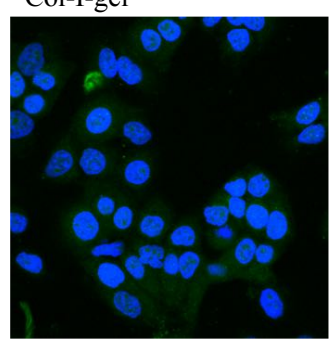

TGF $\beta 1$

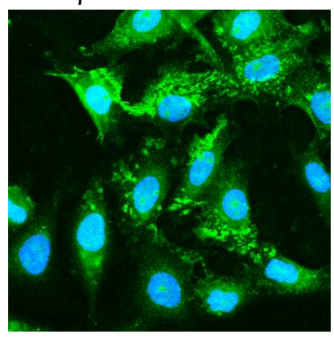

C

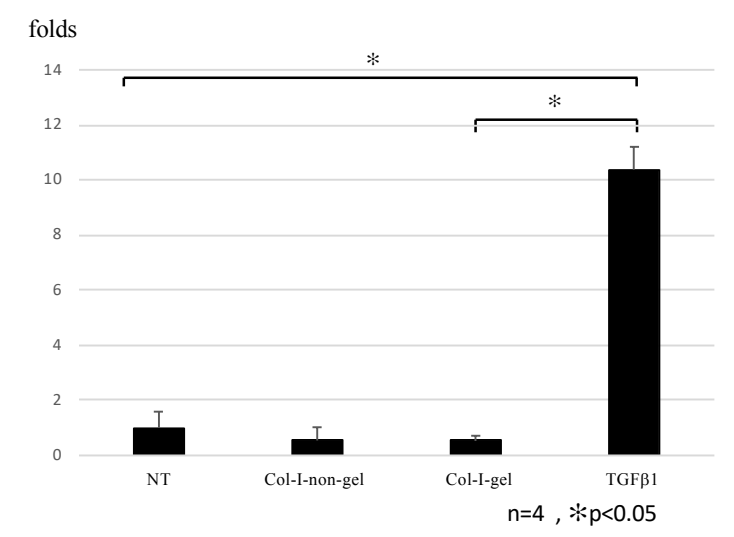

Figure 3. Expression of vimentin in A549 cells. A. A549 cells were cultured on NT, on Col-I-non-gel, on Col-I-gel and with TGF $\beta 1$ for 2 days. Cells were immunostained with anti-vimentin antibody. B. Typical western blot data are shown. C. The levels of protein expression were determined from the western blots and normalized to that of GAPDH. The mean values of vimentin in each condition were divided by that on NT. The results were verified by Student's $t$-test. $* P<0.05$. 

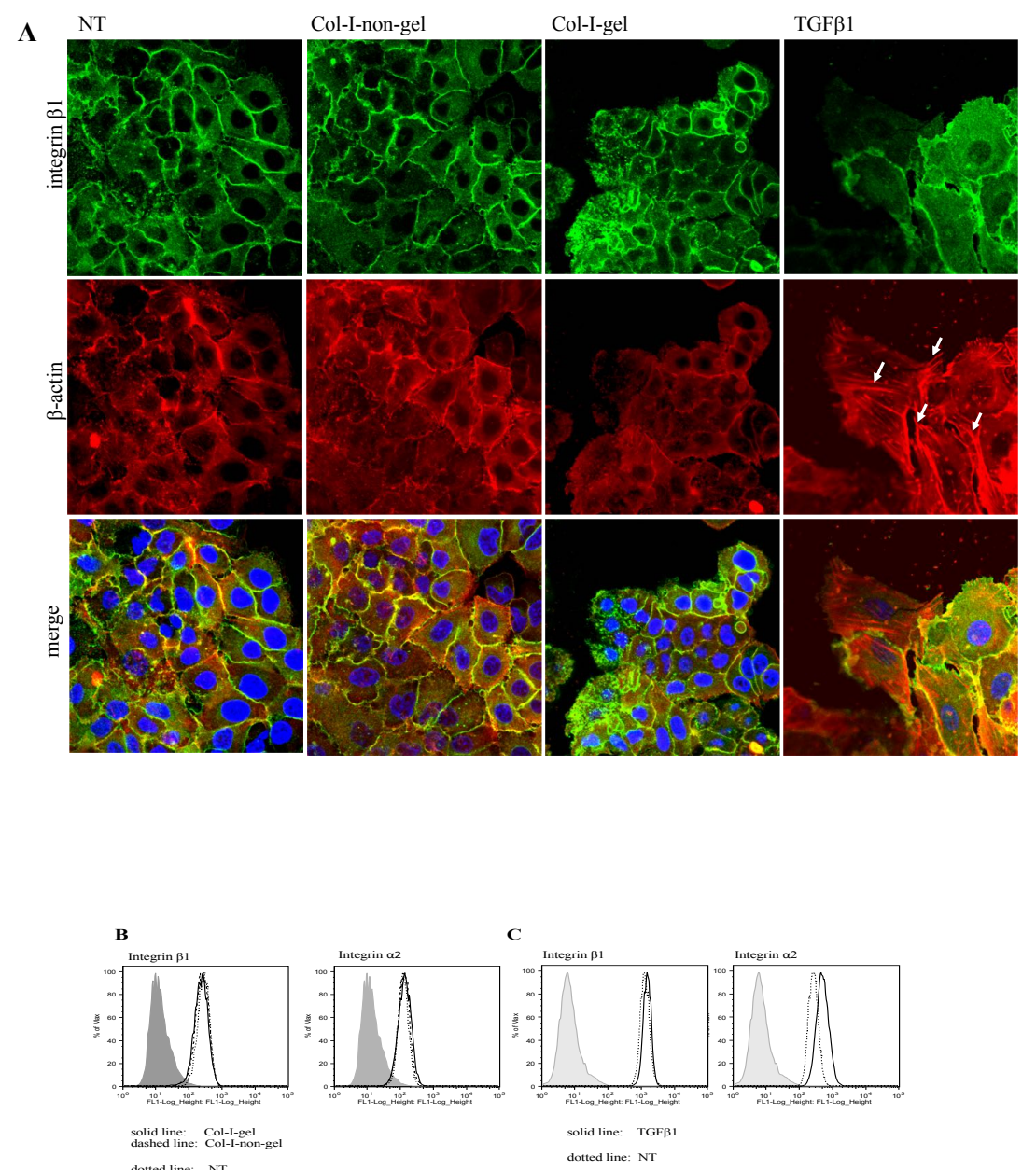

dotted line: NT

solid line: TGFB

Figure 4. Expression of integrins $\beta 1$ and $\alpha 2$ in A549 cells. A. Integrin $\beta 1$ and $\beta$-actin were observed by immunostaining. B. The fluorescence intensity of integrins $\beta 1$ and $\alpha 2$ were estimated by flow cytometric analysis. Comparing with the data on NT, on Col-I-non-gel, and on Col-I-gel. Gray-filled line, unstained cells (negative control); dotted line, on NT; dashed line, on Col-I-non-gel; solid line, on Col-I-gel. C. Comparing with the data on NT and with TGF $\beta 1$. Gray-filled line, unstained cells (negative control); dotted line, NT; solid line, with TGF $\beta 1$. 


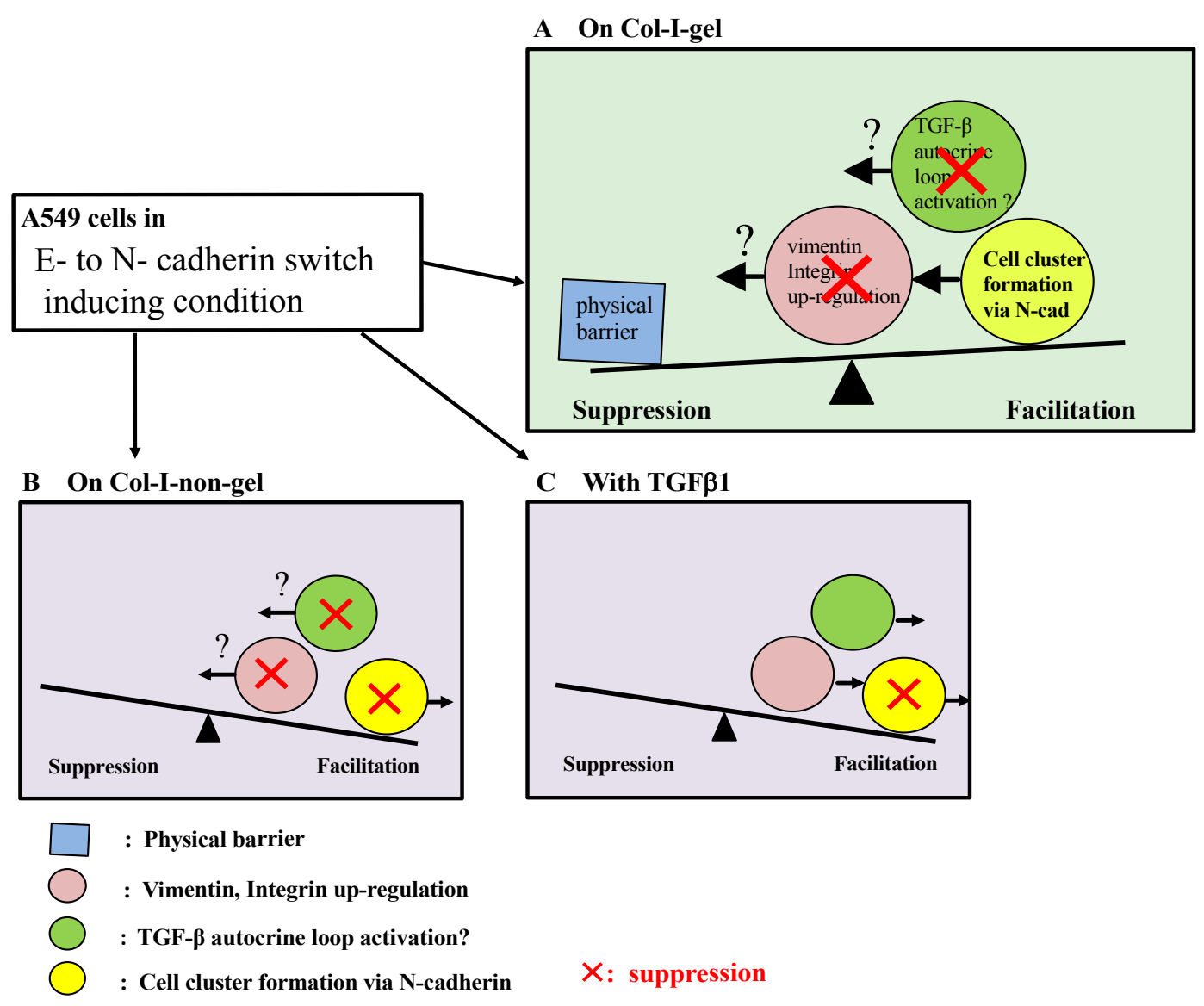

Figure 5. Schematic view of the roles of Col-I fibrous gels on cancer invasion process. A. On Col-I-gel, E- to N- cadherin switching is induced and cell clusters are formed via N-cadherin in A549 cells. The expression levels of mesenchymal intermediate filament (vimentin) and collagen receptor (integrin $\alpha 2 \beta 1$ ) do not increase comparing with on NT. Thus, TGF- $\beta$ autocrine loop might not work. In addition, Col-I fibril lattices might work as a barrier to prevent cell invasion. These changes might work as candidates for suppressor in cancer cell invasion. B. On Col-I-non-gel, unchanged of the expression levels of vimentin and integrin $\alpha 2 \beta 1$, and TGF- $\beta$ autocrine loop suppression work as invasion suppressors. On the other hand, there are neither cell cluster formation via N-cadherin nor barrier of Col-I fibril lattice. $\mathbf{C}$. With TGF $\beta 1$, all of these factors on Col-I-gel do not work. 